\title{
Partially Ordered Constraint Optimization Problems *
}

\author{
Marco Gavanelli \\ Dip. di Ingegneria - University of Ferrara \\ Via Saragat, 1 - 44100 Ferrara, Italy. \\ mgavanelli@ing.unife.it
}

In Constraint Optimization Problems (COP) the objective function induces a total order on the solution set. However, in many real-life applications, more functions, possibly conflicting, should be optimized at the same time, and solutions are ranked by means of a Partial Order.

We propose the model of Partially-Ordered COP, along with a solving algorithm for it:

Definition 1. A Partially-ordered COP (PCOP) is a couple $R=\langle P, f\rangle$, where $P=\left\langle\left\{X_{1}, \ldots, X_{n}\right\},\left\{D_{1}, \ldots, D_{n}\right\}, C\right\rangle$ is a CSP and $f: D_{1} \times \ldots \times D_{n} \mapsto S_{p}$, (where $\left\langle S_{p}, \preceq\right\rangle$ is a partially-ordered set) is a function. A Solution of a PCOP is a solution $S$ of $P$ such that $\nexists S^{\prime}$ solution of $P$ and $f(S) \prec f\left(S^{\prime}\right)$.

The PCOP generalizes many real-life problems, like the Multi-Objective Problem (MOP) or CSPs that embed the concept of partial order on the solution space. E.g., in a MOP, a solution is non-dominated if a better solution (i.e., such that all the objective functions are better) does not exist. In general, there are many non-dominated solutions in a MOP.

A PCOP can be solved in a variety of ways, the most trivial is finding all the solutions of the CSP and a posteriori select only the non-dominated ones. A more efficient approach is an extension of Branch and Bound (B\&B). B\&B is an efficient, widely used method for solving COPs; it could be described as follows: first find a solution (typically using tree search), then add a further unbacktrackable constraint saying that "new solutions must be better than the current best". We have extended B\&B by considering, instead of a single additional constraint, a set of unbacktrackable constraints that limit the next solutions to be better (in the $\preceq$ sense) than the already achieved ones. In other words, to solve a PCOP, we have to store all the solutions of the CSP that are currently believed to be non-dominated.

For example, in the MOP case, the constraints added in the B\&B are $\operatorname{not}(\overline{f(X)} \prec \overline{f(S)})$; i.e., a (tentative) possible solution $X$ will be pruned off if an already obtained solution $S$ is better in all the objective functions: $\forall_{i=1}^{m} f_{i}(X) \leq f_{i}(S)$.

We are currently studying very efficient methods for the propagation of the unbacktrackable constraint store. Experimental results show that our B\&B extension takes about $40-50 \%$ of the time of standard methods providing the whole non-dominated frontier.

* This work was partially supported by CNR

T. Walsh (Ed.): CP 2001, LNCS 2239, p. 763, 2001.

(C) Springer-Verlag Berlin Heidelberg 2001 